Nature Reviews Cardiology | Published online 29 Mar 2018; doi:10.1038/nrcardio.2018.35

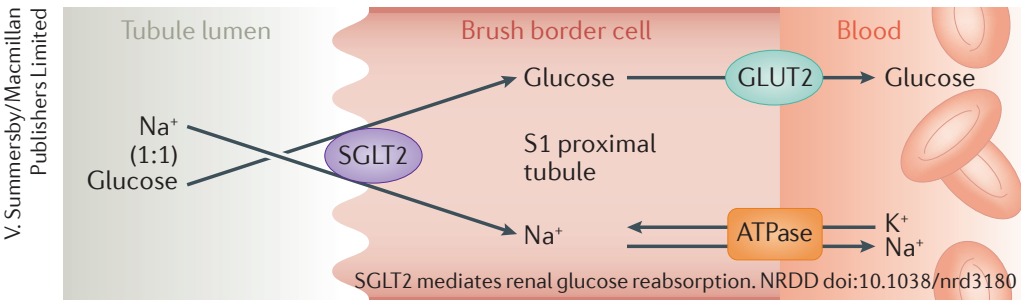

$\Rightarrow$ CARDIOPROTECTION

\section{SGLT2 blockers in T2DM}

Treatment with sodium/glucose cotransporter 2 (SGLT2) inhibitors, which reduce blood glucose levels in patients with type 2 diabetes mellitus (T2DM) by preventing renal reabsorption of glucose, has been linked to a reduced risk of heart failure and cardiovascular death in clinical trials. New results from the CANVAS programme (conducted in 10,142 patients with T2DM and high cardiovascular risk) provide further evidence of these benefits - treatment with the SGLT2 inhibitor canagliflozin led to a $22 \%$ reduction in the composite end point of cardiovascular death or hospitalization for heart failure versus placebo (16.3 versus 20.8 events per 1,000 patient-years). Importantly, patients with pre-existing heart failure seemed to derive more benefit from canagliflozin than did those without heart failure at baseline (HR $0.61,95 \% \mathrm{Cl} 0.46-0.80$ versus HR $0.87,95 \% \mathrm{Cl} 0.72-1.06, P=0.021$ for interaction). "SGLT2 inhibitors should now be prominent in our minds when managing patients with T2DM," asserts Gemma Figtree, lead author on this paper.

The benefits of canagliflozin emerged early during follow-up, suggesting that they are driven by haemodynamic and volume effects leading to preload and afterload reduction. Direct cardiac benefits of SGLT2 inhibition might also result from increased hepatic production of ketone bodies, used as an energy substrate by the failing myocardium. "These beneficial effects of canagliflozin on heart failure outcomes are likely to be accrued on top of those of other therapies for heart failure," comments Figtree.

Complementing the clinical trial data (and building on the CVD-REAL study in European and North American populations), Mikhail Kosiborod et al. report the results of CVD-REAL 2, a study of real-world data on the effects of initiating SGLT2 inhibitors in >400,000 individuals with T2DM from Australia, Canada, Israel, Japan, Singapore, and South Korea. The researchers identified 235,064 episodes of SGLT2 treatment initiation, which were propensity matched to 235,064 treatment initiation episodes for other glucose-lowering agents. During follow-up ( 440,000-490,000 person-years, depending on outcome), initiation of an SGLT2 inhibitor was consistently associated with risk reductions in all-cause death, hospitalization for heart failure, and a composite end point (these two outcomes plus myocardial infarction and stroke) across all countries, all patient subgroups, and in sensitivity analyses. "Our data suggest that the cardiovascular effects of SGLT2 inhibitors extend across ethnic [groups], geographic regions, as well as the cardiovascular risk continuum," Kosiborod observes. "These findings should be reassuring to clinicians."

"From a biological perspective, the results across the board are striking," agrees Figtree. "They are encouraging fundamental researchers to go back to the bench to try to understand the mechanisms involved. Such work is likely to lead to even broader benefits for patients with heart failure."

Caroline Barranco

ORIGINAL ARTICLES Rådholm, K. et al. Canagliflozin and heart failure in type 2 diabetes mellitus: results from the CANVAS program (Canagliflozin Cardiovascular Assessment Study). Circulation https://doi.org/ 10.1161/CIRCULATIONAHA.118.034222 (2018) | Kosiborod, M. et al. Lower cardiovascular risk associated with SGLT-2i in >400,000 patients: the CVD-REAL 2 study. J. Am. Coll. Cardiol. https://doi.org/10.1016/ j.jacc.2018.03.009 (2018)

FURTHER READING Nassif, M. \& Kosiborod, M. Effect of glucose-lowering therapies on heart failure. Nat. Rev. Cardiol. https://doi.org/10.1038/nrcardio.2017.211 (2018)|Chao, E. C. \& Henry, R. R. SGLT2 inhibition - a novel strategy for diabetes treatment. Nat. Rev. Drug Discov. 9, 551-559 (2010)

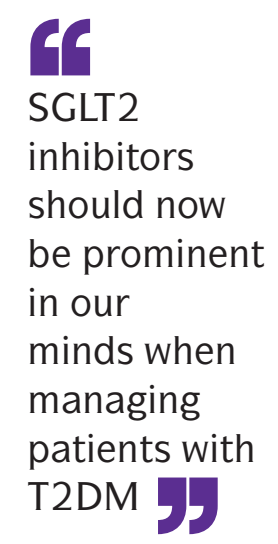

SGLT2

inhibitors

should now

be prominent

in our

minds when

managing

patients with

T2DM 